\title{
Bismuth Containing Quadruple Therapy versus Tailored Therapy as first-line treatments for Helicobacter pylori infection in a high clarithromycin resistance area
}

\section{Byoung Wook Bang}

Inha University College of Medicine

Jong Beom Shin

Inha University College of Medicine

Eun Jung Ko

Inha University College of Medicine

Kye Sook Kwon

Inha University College of Medicine

Yong Woon Shin

Inha University College of Medicine

Young Ju Suh

Inha University College of Medicine

Hyungkil Kim ( $\nabla$ kimhg@inha.ac.kr)

Inha University School of Medicine https://orcid.org/0000-0003-4230-3897

\section{Weonjin Ko}

Inha University College of Medicine

Research article

Keywords: Helicobacter pylori; Clarithromycin; Drug Resistance; Disease eradication

Posted Date: November 5th, 2019

DOI: https://doi.org/10.21203/rs.2.13917/v3

License: (c) (i) This work is licensed under a Creative Commons Attribution 4.0 International License.

Read Full License 


\section{Abstract}

Background Increasing clarithromycin resistance has led to the need for an alternative first-line therapy for the eradication of Helicobacter pylori ( $\mathrm{H}$. pylori) in Korea, and bismuth containing quadruple therapy (BQT) and tailored therapy (TT) have been proposed as alternative regimens. The aim of this study was to compare the eradication rates of BQT and TT as first-line $\mathrm{H}$. pylori eradication therapies. Methods $\mathrm{H}$. pylori infection was diagnosed using the rapid urease test or dual-priming oligonucleotide-based multiplex polymerase chain reaction (DPO-PCR) during endoscopy. Patients positive for $\mathrm{H}$. pylori were divided into two groups; those tested using the rapid urease test received empirical BQT (the BQT group) whereas those tested by DPO-PCR received TT (the TT group). Eradication rates, adverse events, and overall medical costs, which included diagnostic test and eradication regimen costs, were compared. Results Three hundred and sixty patients were included in the study (TT group 178, BQT group 182). The modified intention-to-treat eradication rates of BQT and TT were $88.2 \%(142 / 161)$ and $80.3 \%(118 / 147)$, respectively $(p=0.055)$, and corresponding eradication rates in the per-protocol population were $88.8 \%$ $(142 / 160)$ and $81.4 \%(118 / 145)(p=0.07)$. Compliance and adverse event rates were similar in the two groups. Average medical costs were $\$ 90.3$ per patient in the TT group and $\$ 75.5$ in the BQT group $(p=0.000)$. Conclusions Empirical BQT and tailored therapy were similar in terms of $\mathrm{H}$. pylori eradication rate, safety, and tolerability, but BQT was more cost-effective.

\section{Background}

Helicobacter pylori (H. pylori) is the major cause of chronic gastritis, peptic ulcer disease, and gastric cancer. Thus, the eradication of $H$. pylori infections dramatically decreases peptic ulcer recurrence and prevents gastric cancer. ${ }^{1}$ Clarithromycin based triple therapy regimen consisting of a proton pump inhibitor (PPI), amoxicillin, and clarithromycin for 7 days are globally accepted first-line treatment for the eradication of $H$. pylori, ${ }^{2}$ but eradication rates associated with clarithromycin based triple therapy have declined to unacceptable levels and clarithromycin resistance has been reported to be the main reason for eradication failure. Therefore, new therapeutic regimens are needed in areas of high clarithromycin resistance. $^{3}$

The Maastricht $\mathrm{V}$ consensus recommended clarithromycin susceptibility testing when considering clarithromycin based triple therapy as a first-line treatment, except in areas with low clarithromycin resistance rates $(<15 \%)^{4}$. On the other hand, in areas with high clarithromycin resistance rates, bismuth based quadruple therapy (BQT; bismuth subsalicylate, tetracycline, metronidazole, and PPI) has been recommended as a first-line empirical treatment because its efficacy is irrelevant to clarithromycin resistance. ${ }^{4-6}$ In Korea, the $3^{\text {rd }}$ Clinical Practice Guideline for H. pylori published in 2013 recommended clarithromycin based triple therapy as the first-line therapy, despite falling eradication rates, and also that BQT be considered as the first-line regimen in areas of high clarithromycin resistance ${ }^{6}$. In Korea, clarithromycin resistance varies regionally from $8.5 \%$ to $37.5 \%$ and has been increasing over recent 
years. ${ }^{7-9}$ Accordingly, testing for clarithromycin resistance is becoming increasingly important prior to treatment decision making.

Clarithromycin acts by binding to $23 \mathrm{~S}$ ribosomal RNA (rRNA), and thus, inhibiting protein synthesis, but cannot bind to $23 S$ rRNA harboring a point mutation. ${ }^{10}$ In most cases, resistance to clarithromycin is caused by point mutations at the 2142 or 2143 positions of the $23 S$ rRNA gene. Dual-priming oligonucleotide-based multiplex polymerase chain reaction (DPO-PCR) can be used to detect clarithromycin resistance as well as $H$. pylori, and unlike conventional PCR, DPO-PCR blocks nonspecific binding sites and suppresses imperfect primer annealing, and thus, increases sensitivity and specificity. ${ }^{11,12}$ In addition, testing is straightforward and rapid, and thus, DPO-PCR test enables tailored therapy (TT) without $H$. pylori culture.

Bismuth based quadruple therapy is a recognized, effective $2^{\text {nd }}$-line therapy for $H$. pylori according to domestic and international practice guidelines $5,6,13,14$. According to one meta-analysis of primarily western studies, the eradication rate of BQT used as a first-line treatment was $77.6 \%$ by ITT analysis. ${ }^{15}$ However, recent studies on the efficacy of BQT as a first-line treatment are lacking. The aim of this study was to compare the efficacies of first-line BQT and TT for the treatment of H. pylori, and its secondary aim was to compare their safeties and cost-effectivenesses. In addition, we also evaluated the efficacy of levofloxacin-based therapy as a second-line treatment after BQT or TT failure.

\section{Methods}

\section{Patients and study design}

Nine hundred and fifty seven patients that underwent upper gastrointestinal endoscopy at Inha University hospital and testing for $H$. pylori from August 2016 to April 2018 were prospectively screened. After exclusions, patients were assigned to one of two groups using a random number generator. For $\mathrm{H}$. pylori testing, one group was tested using a rapid urease test (RUT), whereas the other group was tested by DPO-PCR. Of those found to be $H$. pylori positive, those tested by RUT received empirical BQT and those tested by DPO-PCR received tailored therapy (TT). Patients that had previously received $H$. pylori eradication therapy and those with a severe illness, such as liver cirrhosis, chronic kidney disease, or heart failure, were excluded, as were pregnant or possibly pregnant patients. Eradication success rates, adverse events related to medications, and compliances with treatments were investigated and compared. All patients provided informed consent, and the study was approved by the Institutional Review Board of Inha University (2016-01-21) and registered as a trial at ClinicalTrials.gov (NCT03361267).

\section{Test for H. pylori}

Two gastric tissue specimens were obtained from antrum and corpus for rapid urease testing (RUT; Bio Helicobacter Test, Shinsung Pharmaceutical Co., Ltd, Suwon, Korea) or DPO-PCR. RUT status was 
assessed within 24 hours of sampling. DPO-PCR was performed using Seeplex ${ }^{\circledR}$ H. pylori-ClaR ACE Detection kits (Seegene Inc., Seoul). This test can detect the A2142G and A2143G mutations in 23S rRNA. If two-point mutations were of the wild type, $\mathrm{H}$. pylori was considered susceptible, but if one of the twopoint mutations was present, clarithromycin resistance was deemed positive.

\section{Treatment for $\boldsymbol{H}$. pylori}

In the BQT group, the BQT regimen was prescribed for 7 days. This regimen consisted of rabeprazole 20 $\mathrm{mg}$ twice a day, metronidazole $500 \mathrm{mg}$ three times a day; bismuth $300 \mathrm{mg}$ four times a day, and tetracycline $500 \mathrm{mg}$ four times a day for 7 days. In the TT group, clarithromycin triple therapy was prescribed in patients with clarithromycin-sensitive $H$. pylori, and BQT was prescribed in patients with clarithromycin-resistant $H$. pylori for 7 days. Clarithromycin triple therapy consisted of rabeprazole $20 \mathrm{mg}$ twice a day, amoxicillin 1,000 mg twice a day and clarithromycin $500 \mathrm{mg}$ twice a day for 7 days. Patients that remained positive after first-line therapy were retreated with a levofloxacin based regimen, which consisted of rabeprazole $20 \mathrm{mg}$ twice daily, amoxicillin 1,000 mg twice daily, and levofloxacin 500mg once daily for 7 days.

\section{Follow-up and outcomes}

Patients returned to our clinics within 2 weeks after commencing drug administration to assess drug compliance and adverse effects, which were assessed using a self-report questionnaire. Non-compliance

was defined as taking $<90 \%$ of the prescribed study drug. H. pylori eradication was confirmed by ${ }^{13} \mathrm{C}$-urea breath testing (UBT; Korea Otsuka Pharmaceutical Co., Ltd, Seoul) at least 4 weeks after completing eradication therapy. The cut-off value used for the ${ }^{13} \mathrm{C}$-urea breath test was $2.5 \%$.

\section{Medical costs}

To assess cost-effectiveness, the two groups were compared with respect to costs of diagnostic tools and of the eradication regimen, excluding common costs (e.g., endoscopy and biopsy). The cost of the rapid urease test was $\$ 11.7$, and that of DPO-PCR was $\$ 33.6$, and the costs of the seven-day clarithromycin triple regimen and of the seven-day Bismuth quadruple regimen were $\$ 32.0$ and $\$ 24.2$, respectively. The cost of the urea breath test (used for eradication confirmation) was $\$ 22.6$, and of the seven-day levofloxacin based triple therapy was $\$ 25.6$.

\section{Sample size calculation}


Based on a review of previous studies, the eradication rates of TT and BQT were assumed to be $91 \%$ and $78 \%{ }^{8,15}$ Sample size was calculated based on a non-inferiority trial at a both-sided alpha significance level of 0.05 , a power of 0.90 , and a non-inferiority margin of 0.13 .

$n=\frac{\left(z_{\alpha / 2}+z_{\beta}\right)^{2}\left(p_{c} q_{c}+p_{t} q_{t}\right)}{\left(p_{c}-p_{t}\right)^{2}}$

The calculated sample size was 162 per group, and thus, assuming a dropout rate of $10 \%$, at least 180 patients were required per group.

\section{Statistical analysis}

The primary study end point was $H$. pylori eradication rate, which was evaluated by intention-to-treat (ITT), modified intention-to-treat (mITT), and per protocol (PP) analyses. The ITT analysis included all enrolled patients who received an eradication drug, and mITT analysis included patients who took at least one dose of the prescribed drugs and received UBT after eradication therapy. PP analysis was conducted after excluding patients who took $<90 \%$ of the prescribed drugs, those that did not return for follow up, and those that violated the study protocol. The secondary end points were frequency of adverse events, drug compliance, and medical cost. $P$-values of $<0.05$ were considered statistically significant, and the analysis was conducted using SPSS ver. 19 for Windows (SPSS Inc. Chicago, IL, USA).

\section{Results}

\section{Demographics of the study groups}

A flow chart of patients that received either of the two treatment regimens is provided in Figure 1.957 patients were initially screened for $H$. pylori infection by RUT or DPO-PCR, and 493 (51.5\%) were positive. Of these, 133 were excluded because they refused treatment or to participate in the study. The remaining 360 patients were allocated to the BQT or TT group according to diagnostic method. Demographic and clinical characteristics are summarized in Table 1 and were not significantly different in the two groups.

\section{Outcomes of BQT and TT}

ITT eradication rates in the BQT and TT groups were $78.0 \%$ and $66.3 \%$ respectively. Corresponding $\mathrm{mITT}$ and PP eradication rates were $88.2 \%$ and $80.3 \%$ and $88.8 \%$ and $81.4 \%$, respectively (Table 2 ). 


\section{Patient compliance and adverse events}

Compliance was assessed for 308 patients that completed treatment and was no different in the two groups $(98.6 \%$ (145/147) in the TT group and 99.4\% (160/161) in the BQT group. Most patients did not experience a drug-related adverse or minor side effect (Table 3). Two patients discontinued drugs due to adverse effects (nausea and diarrhea), but recommenced treatment after symptoms improved.

\section{TT group}

The clarithromycin resistance rate in the TT group was $21.9 \%$ (39/178). The A2142G mutation was detected in one patient $(2.6 \%, 1 / 39)$, while the A2143G mutation was detected in $37(94.9 \%, 37 / 39)$. Both mutations were detected in one patient $(2.6 \%, 1 / 39)$. For the 139 patients with clarithromycin-sensitive $H$. pylori, the eradication rate of clarithromycin triple therapy was $79.0 \%$ by $\mathrm{mITT}$ analysis and $79.7 \%$ by PP analysis. On the other hand, for the 39 patients with clarithromycin-resistant $H$. pylori, corresponding eradication rates for BQT were $89.3 \%$ and $88.8 \%$ respectively.

\section{Levofloxacin triple therapy}

In this study, 40 patients (25 patients in the TT group and 15 patients in the BQT group) who failed firstline therapy were treated with second-line levofloxacin based triple therapy. Twenty-one of these 40 patients achieved eradication, but 13 failed second-line eradication. Six of the 40 patients were not followed up. Eradication rates for levofloxacin triple therapy as determined by ITT and PP analysis were $52.5 \%$ and $61.8 \%$, respectively.

\section{Medical cost}

Average medical costs were calculated by summing the costs of diagnosis and treatment for first-line eradication treatment and by assuming patients that failed first-line therapy received second-line therapy. Average medical costs per patient were then $\$ 90.3$ and $\$ 75.5$ for the TT and BQT groups, respectively $(p=0.000)$.

\section{Discussion}

In Korea, clarithromycin resistance varies regionally, and therefore, the efficacies of $H$. pylori eradication regimens depend on areas. Thus, we sought to determine the prevalence of clarithromycin resistance in the Incheon area, which abuts western Seoul. In this region the clarithromycin resistance rate was previously reported to be $37.4 \%$, but it was included patients who were eradicated $H$. pylori infection, and 
thus, clarithromycin resistance may have been overestimated. ${ }^{16}$ In the present study, clarithromycin resistance was $21.9 \%(39 / 178)$. This result indicates Incheon is a region of high clarithromycin resistance and that empirical clarithromycin triple therapy is not a suitable first-line regimen for $H$. pylori eradication in the region.

According to the Maastricht $V$ guidelines, in a setting with high clarithromycin resistance $(>15 \%)$, choice of therapy should be based on the frequencies of metronidazole and of combined clarithromycin and metronidazole resistance. However, antimicrobial susceptibility testing by culture and antibiogram is difficult and time-consuming, and not available in all institutions. In addition, the success rate of $H$. pylori culture has been reported relatively low at $55-73 \% .{ }^{17}$ Furthermore, if empirical bismuth-based quadruple therapy is expected to be used, it is not advisable to perform antimicrobial susceptibility testing because the risk of tetracycline resistant strains is extremely low and metronidazole resistance has little effect on eradiation rate. ${ }^{18}$ Therefore, empirical BQT provides a good alternative first-line eradication therapy in areas of high clarithromycin resistance.

Based on the results of previous studies, we considered tailored therapy would have a higher eradication rate than empirical BQT. ${ }^{8,15}$ However, we found the eradication rates of BQT and TT were not significantly different (88.8 vs $81.4, p=0.07)$. In particular, in patients with clarithromycin-sensitive $H$. pylori in the TT group, the eradication rate of clarithromycin triple therapy was only $79.4 \%(94 / 118)$, which was lower than that previously reported in a Korean study. ${ }^{8}$ Although DPO-PCR can diagnose A2142G and A2143G variants, it does not provide a comprehensive view of clarithromycin resistance and cannot identify resistance to other antibiotics. Nevertheless, point mutation of $23 \mathrm{~S}$ rRNA, which usually involves the substitution of guanine with adenine at nucleotides 2142 and 2143 is detected by DPO-PCR and is known to be associated with clarithromycin resistance. Substitution of adenine with cytosine at position 2142 is also less frequently observed. ${ }^{19}$ Other point mutations in $23 S$ rRNA, such as of A2115G, G2141A, T2117C, T2182C, T2289C, G224A, C2245T and C2611A, have also been reported, but their clinical significances are unknown. Macrolide resistance caused by the efflux pump system also plays an important role in promoting multidrug $H$. pylori resistance. ${ }^{19}$ Thus, despite the absence of A2142G or A2143G mutations, it can be inferred the eradication rate was lower than expected in the present study because of other point mutations or the involvements of efflux pump systems.

Maastricht $\mathrm{V}$ guideline suggests that the eradication rate should be over $80 \%$ by ITT analysis and over $90 \%$ by PP analysis. In our study, we use only BQT therapy and clarithromycin based triple therapy as a first line therapy. So the eradication rate was below the Maastricht $V$ guideline. We should study further to improve eradication rate, and efficacy in aspect to complication and medical cost. And recently, fluoroquinolone resistance has increased in our region. In the present study, RUT was used in the BQT group and DPO-PCR in the TT group to diagnose H. pylori infection, and thus, $H$. pylori diagnostic rates may have differed in the two groups. Originally, we intended to test all participants using RUT and DPOPCR, regardless of treatment regimen. However, our IRB committee determined that it was unethical to select an eradication regimen irrespective of clarithromycin resistance results, and thus, we modified the 
study design. Rapid urease testing is reliable and its sensitivity and specificity are $90 \%$ and $95-100 \% 20,21$. False-negatives are more common than false positives, and thus, negative results do not exclude the possibility of $H$. pylori infection, but false positive are uncommon. The Seeplex ${ }^{\circledR}$ ClaR-H. pylori ACE detection kit has been reported to have a sensitivity of $97.7 \%$ and a specificity of $83.1 \%$ for the diagnosis of $H$. pylori versus culture testing, ${ }^{22}$ and thus, our diagnostic method is not likely to have biased diagnosis or eradication rates due to false positive results.

In this study, levofloxacin-based triple therapy was used as second-line therapy following failed first-line treatment. In the Maastricht $V$ consensus, fluoroquinolone-containing triple therapy, such as levofloxacin, is recommended as second-line treatment after failure of first-line clarithromycin-based and second-line bismuth quadruple therapy. According to a meta-analysis, the eradication rate of levofloxacin-based triple therapy as a second-line treatment was $74.8-79.6 \% .{ }^{23}$ In the present study, the eradication rate of levofloxacin-based triple therapy was $61.8 \%$ by PP analysis, which is lower rate than those reported previously. This may have been related to a rapid increase in fluoroquinolone resistance over recent years. A recent Korean study reported a resistance rate for levofloxacin of $5.7 \%$ for $2003-2005$, but a rate of $34.6 \%$ for 2009-2012. ${ }^{24}$ This rapid increase in levofloxacin resistance may be due to cross-resistance to other fluoroquinolones such as ciprofloxacin or moxifloxacin. Also, BQT is recommended as $2^{\text {nd }}$ line eradication regimen after failure of triple therapy. But, in this study, patients took levofloxacin based triple therapy after failure of clarithromycin based triple therapy. In our study, we tried to compare the compliance and medical cost between BQT and TT group. So we used same $2^{\text {nd }}$ line regimen in failure group.

According to recently published guidelines, the recommended duration for $H$. pylori eradication therapy is 14 days, ${ }^{4,14}$ whereas we used a 7-day regimen. When we planned this study, the 14 -day regimen was not recommended because the eradication rates of 7- and 14-day regimens were believed to be similar, and thus, Korean medical insurance only covered 7 day regimens. However, metronidazole resistance can be overcome by long-duration treatment. Therefore, the eradication success rate of BQT can be improved after 14 days treatment of BQT.

In terms of medical cost, the rapid urease test was less expensive than the DPO-PCR in diagnostic test, and the 7-day BQT regimen was less expensive than the 7-day clarithromycin triple therapy regimen, and thus, based on diagnostic and therapeutic costs, empirical BQT was preferred. However, in a recent study, conducted in Incheon city, it was reported that DPO-PCR guided target therapy was equivalent or superior to empirical clarithromycin triple therapy in terms of cost effectiveness. ${ }^{16}$

Our research has several limitations. First, it was conducted at a single center in Incheon, and thus, it is difficult to generalize our results. Second, as mentioned above, different $H$. pylori diagnostic methods were used in the two groups, and thus, group diagnosis rates may have different results. However, since the sensitivities of both diagnostic methods are high, we believe it is unlikely that this greatly influenced our results. Third, $H$. pylori culture along with real antibiotic resistance test had not been performed. And 
validation study for DPO-PCR was not performed in our institution. Fourth, we think because of small sample size, BQT showed somewhat higher eradication rate than TT in despite of the other previous study.

\section{Conclusions}

In a high clarithromycin resistance area (Incheon), the H. pylori eradication rates, safeties, and tolerabilities of tailored therapy and BQT were similar, but overall empirical BQT was preferred over tailored therapy because of its cost-effectiveness.

\section{Abbreviations}

H. pylori: Helicobacter pylori, BQT: Bismuth containing quadruple therapy, TT: Tailored therapy, DPO-PCR: Dual-priming oligonucleotide-based multiplex polymerase chain reaction, PPI: Proton pump inhibitor, BQT: Bismuth quadruple therapy, rRNA: Ribosomal RNA, RUT: Rapid urease test, UBT: ${ }^{13} \mathrm{C}$-urea breath testing, ITT: Intention-to-treat, mITT: Modified intention-to-treat, PP: Per protocol

\section{Declarations}

\section{Acknowledgement}

The authors thank the study participants, without whom the study would never have been accomplished.

\section{Author's contributions}

HK: Principal investigator, who contributed to the drafting and critical revision of the manuscript for important intellectual content. BW: study design, acquisition of data, analysis and interpretation of data. JB, EJ, WJ, KS, YW, YJ: study conception and design and to the acquisition, analysis, and interpretation of data. All authors have read and approved the manuscript.

\section{Funding}

This research was supported by INHA UNIVERSITY Research Grant. Also, it was partially supported by grant from the Incheon Medical Association.

\section{Availability of data and materials}

All data generated or analyzed during this study are included in this published article and its supplementary information files.

\section{Ethics approval and consent to participate}

This study was conducted in accordance with the ethical principles originating in the Declaration of Helsinki and the international Conference on Harmonization Good Clinical Practice guidelines. All 
patients provided informed consent by written, and the study was approved by the Institutional Review Board of Inha University (2016-01-21) and registered as a trial at ClinicalTrials.gov (NCT03361267).

\section{Competing interests}

This research was supported by INHA UNIVERSITY Research Grant. Also, it was partially supported by grant from the Incheon Medical Association. The funding body had a role in data analysis and interpretation. No other competing interests exist.

\section{Consent for publication}

Not applicable.

\section{Author details}

${ }^{1}$ Division of Gastroenterology, Department of Internal Medicine, Inha University College Medicine, Incheon, Republic of Korea

${ }^{2}$ Department of Biostatistics, Inha University College of Medicine, Incheon, Republic of Korea

\section{References}

1. McColl KE. Helicobacter pylori infection. N Engl J Med. 2010;362:1597-1604.

2. Malfertheiner P, Megraud F, O'Morain CA, et al. Management of helicobacter pylori infection--the Maastricht IV/ Florence consensus report. Gut. 2012;61:646-664.

3. Graham DY, Fischbach L. Helicobacter pylori treatment in the era of increasing antibiotic resistance. Gut. 2010;59:1143-1153.

4. Malfertheiner P, Megraud F, O'Morain CA, et al. Management of helicobacter pylori infection-the Maastricht V/Florence consensus report. Gut. 2017;66:6-30.

5. Chey WD, Leontiadis GI, Howden CW, Moss SF. ACG clinical guideline: Treatment of helicobacter pylori infection. Am J Gastroenterol. 2017;112:212.

6. Kim SG, Jung H, Lee HL, et al. Guidelines for the diagnosis and treatment of Helicobacter pylori infection in Korea, 2013 revised edition. J Gastroenterol Hepatol. 2014;29:1371-1386.

7. Woo H, Park DI, Park H, et al. Dual-priming oligonucleotide-based multiplex PCR for the detection of helicobacter pylori and determination of clarithromycin resistance with gastric biopsy specimens. Helicobacter. 2009;14:22-28.

8. Lee HJ, Kim JI, Cheung DY, et al. Eradication of helicobacter pylori according to $23 \mathrm{~S}$ ribosomal RNA point mutations associated with clarithromycin resistance. J Infect Dis. 2013;208:1123-1130.

9. Lee JY, Kim N, Kim MS, et al. Factors affecting first-line triple therapy of helicobacter pylori including CYP2C19 genotype and antibiotic resistance. Dig Dis Sci. 2014;59:1235-1243. 
10. Occhialini A, Urdaci M, Doucet-Populaire F, Bebear CM, Lamouliatte H, Megraud F. Macrolide resistance in helicobacter pylori: Rapid detection of point mutations and assays of macrolide binding to ribosomes. Antimicrob Agents Chemother. 1997;41:2724-2728.

11. Yoon K, Park SW, Lee SW, Kim BJ, Kim JG. Clarithromycin-based standard triple therapy can still be effective for helicobacter pylori eradication in some parts of the korea. J Korean Med Sci. 2014;29:1240-1246.

12. Lee JW, Kim N, Kim JM, et al. Prevalence of primary and secondary antimicrobial resistance of helicobacter pylori in Korea from 2003 through 2012. Helicobacter. 2013;18:206-214.

13. Lee SW, Kim HJ, Kim JG. Treatment of helicobacter pylori infection in Korea: A systematic review and meta-analysis. J Korean Med Sci. 2015;30:1001-1009.

14. Fallone CA, Chiba N, van Zanten SV, et al. The Toronto consensus for the treatment of helicobacter pylori infection in adults. Gastroenterology. 2016;151:51-69. e14.

15. Venerito M, Krieger T, Ecker T, Leandro G, Malfertheiner P. Meta-analysis of bismuth quadruple therapy versus clarithromycin triple therapy for empiric primary treatment of helicobacter pylori infection. Digestion. 2013;88:33-45.

16. Gweon TG, Kim JS, Kim BW. An economic modeling study of helicobacter pylori eradication: Comparison of dual priming oligonucleotide-based multiplex polymerase chain reaction and empirical treatment. Gut Liver. 2018;12:648-654.

17. Smith SM, O'Morain C, McNamara D. Antimicrobial susceptibility testing for helicobacter pylori in times of increasing antibiotic resistance. World J Gastroenterol. 2014;20:9912-9921.

18. Malfertheiner P, Bazzoli F, Delchier J. Helicobacter pylori eradication with a capsule containing bismuth subcitrate potassium, metronidazole, and tetracycline given with omeprazole versus clarithromycin-based triple therapy: A randomised, open-label, non-inferiority, phase 3 trial (vol 377, pg 905, 2011). Lancet. 2011;378:1778-1778.

19. Francesco VD, Zullo A, Hassan C, Giorgio F, Rosania R, lerardi E. Mechanisms of helicobacter pylori antibiotic resistance: An updated appraisal. World J Gastrointest Pathophysiol. 2011;2:35-41.

20. El-Zimaity HM, Al-Assi MT, Genta RM, Graham DY. Confirmation of successful therapy of helicobacter pylori infection: Number and site of biopsies or a rapid urease test. Am J Gastroenterol. 1995;90.

21. Woo JS, Hala M, Genta RM, Yousfi MM, Graham DY. The best gastric site for obtaining a positive rapid urease test. Helicobacter. 1996;1:256-259.

22. Nishizawa T, Suzuki H. Mechanisms of helicobacter pylori antibiotic resistance and molecular testing. Frontiers in molecular biosciences. 2014;1:19.

23. Chen P, Wu M, Chen $C$, et al. Systematic review with meta-analysis: The efficacy of levofloxacin triple therapy as the first-or second-line treatments of helicobacter pylori infection. Aliment Pharmacol Ther. 2016;44:427-437.

24. Lee JW, Kim N, Kim JM, et al. Prevalence of primary and secondary antimicrobial resistance of Helicobacter pylori in Korea from 2003 through 2012. Helicobacter. 2013;18:206-214. 


\section{Tables}

Table 1 Basic characteristics of patients in the two study groups

\begin{tabular}{lccccc}
\hline & TT group & BQT group & p-value \\
\hline No. of patients & 178 & 182 & \\
\hline Male gender, n (\%) & 125 & $(70.22)$ & 110 & $(60.44)$ & 0.051 \\
\hline Age, mean \pm SD (years) & 56.9 & $(12.52)$ & 57.9 & $(11.59)$ & 0.670 \\
\hline Indication, n (\%) & & & & & \\
Peptic ulcer & 62 & $(34.83)$ & 72 & $(39.56)$ & 0.445 \\
Stomach cancer & 46 & $(25.84)$ & 32 & $(17.58)$ & \\
Gastric adenoma & 23 & $(12.92)$ & 23 & $(12.63)$ & \\
Gastritis & 40 & $(22.47)$ & 46 & $(25.27)$ & \\
Functional dyspepsia & 7 & $(3.93)$ & 8 & $(3.39)$ & \\
\hline
\end{tabular}

TT, tailored therapy; BQT, Bismuth quadruple therapy.

Table 2. Helicobacter pylori eradication efficacies in the two groups

\begin{tabular}{lccccc}
\hline & \multicolumn{2}{c}{ TT group } & \multicolumn{2}{c}{ BQT group } & p-value \\
\hline ITT eradication rates, n (\%) & $118 / 178$ & $(66.3)$ & $142 / 182$ & $(78.0)$ & 0.013 \\
Modified ITT eradication rates, n (\%) & $118 / 147$ & $(80.3)$ & $142 / 161$ & $(88.2)$ & 0.055 \\
PP eradication rates, n (\%) & $118 / 145$ & $(81.4)$ & $142 / 160$ & $(88.8)$ & 0.067 \\
\hline
\end{tabular}

TT, tailored therapy; BQT, Bismuth quadruple therapy; ITT, intention-to-treat; mITT, modified intention-to-treat; PP, per protocol. 
Table 3. Compliance and adverse events in the two groups

\begin{tabular}{lrrrrr}
\hline & \multicolumn{2}{c}{ TT group } & BQT group & p-value \\
\hline Compliance, n (\%) & $145 / 147$ & $(98.6)$ & $160 / 161$ & $(99.4)$ & 0.509 \\
\hline Adverse events, n (\%) & & & & & \\
None & 136 & $(93.2)$ & 145 & $(90.1)$ & 0.564 \\
Bitter taste & 5 & $(3.42)$ & 7 & $(4.35)$ & \\
Nausea/vomiting & 3 & $(2.05)$ & 3 & $(1.86)$ \\
Diarrhea & 2 & $(1.37)$ & 2 & $(1.24)$ \\
Headache & 0 & $(0.00)$ & 1 & $(0.62)$ \\
Others & 0 & $(0.00)$ & 3 & $(1.86)$ & \\
\hline
\end{tabular}

Figures 


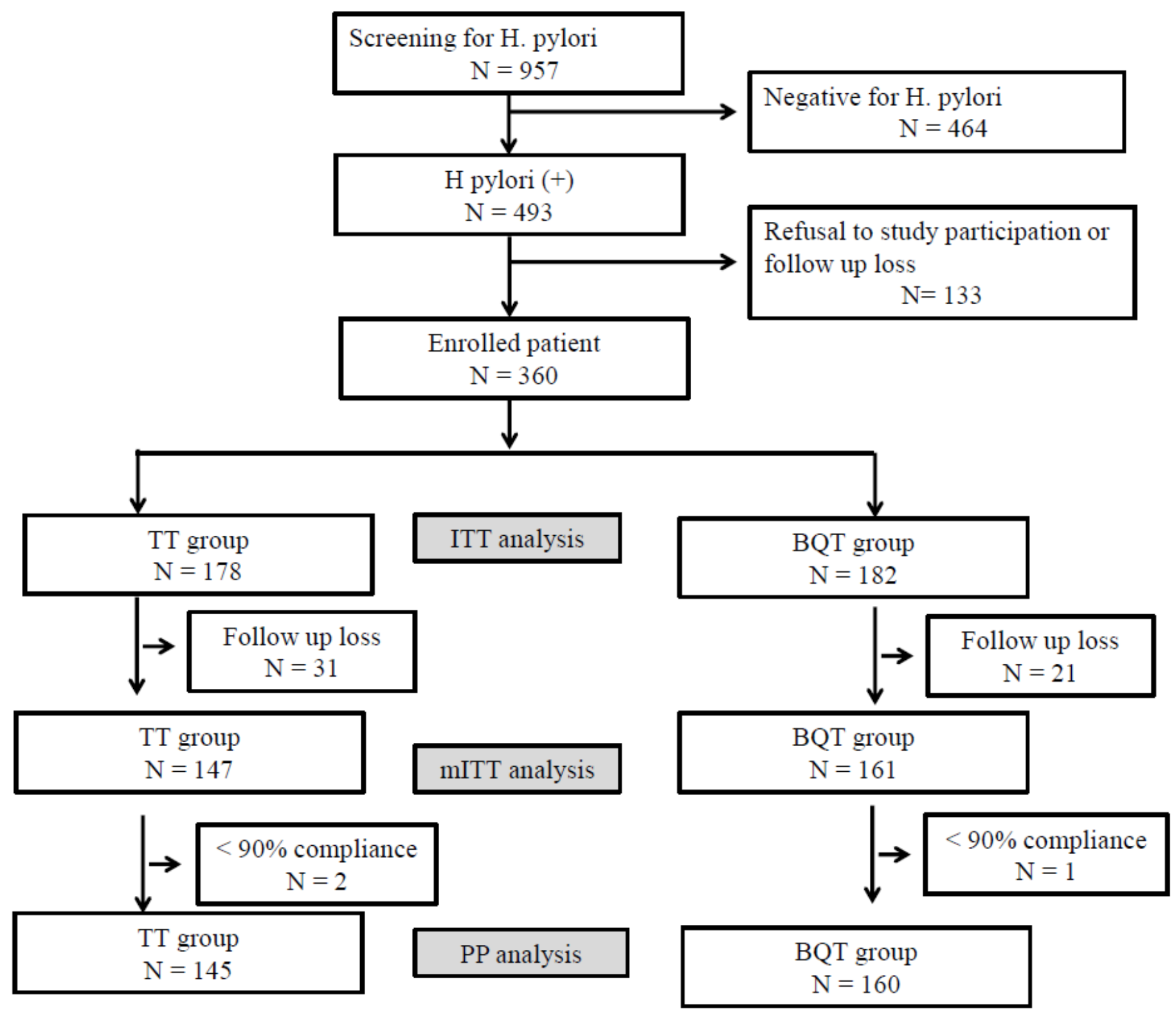

Figure 1

Study flowchart. TT, tailored therapy; BQT, Bismuth quadruple therapy; ITT, intention-to-treat; mITT, modified intention-to-treat; PP, per protocol. 


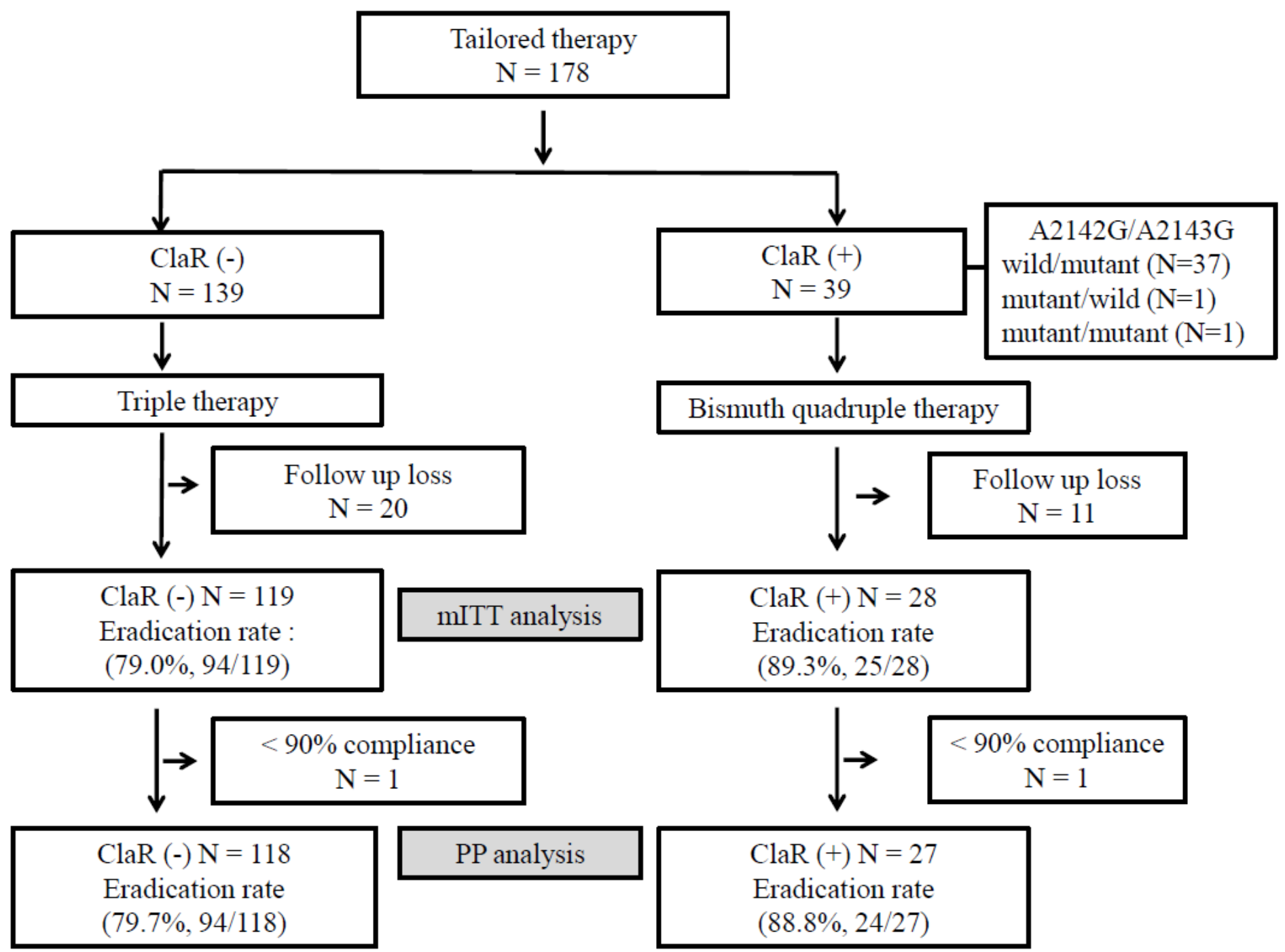

Figure 2

Flowchart of patients in the tailored therapy group. TT, tailored therapy; ITT, intention-to-treat; mITT, modified intention-to-treat; PP, per protocol; BQT, Bismuth quadruple therapy.

\section{Supplementary Files}

This is a list of supplementary files associated with this preprint. Click to download.

- CONSORTChecklist.pdf 